\title{
Making in The Colonias: Motivating STEM Participation through a Making as Micro-Manufacturing Model
}

\section{Mr. Osazuwa John Okundaye Jr, Texas A\&M University}

Osazuwa is a first year PhD student at Texas A\&M University. He is a part of the Texas A\&M Embodied Interaction Lab (TEILab). His research is motivated by the idea of an embodied conception of the mind. He comes from an interdisciplinary background having earned a Bachelor's degree in psychology and a Masters of Science in Visualization afterward. He is versed in engaging the theoretical aspects of HumanComputer Interaction while able to engage in computer graphics applications (computer-aided design, modeling, animation, and 3D fabrication) and concepts pertaining to Computer Science.

\section{Dr. Malini Natarajarathinam, Texas A\&M University}

Dr. Malini Natarajarathinam joined the faculty of Industrial Distribution Program at Texas A\&M University in 2007. Natarajarathinam received her Ph.D. in Supply Chain Management from The University of Alabama. She received her Bachelor of Engineering (Major: Industrial and Systems Engineering) from Anna University [Tamilnadu, India], her MS in Industrial Engineering from Auburn University, her MA in Management Science and MS in Applied Statistics from The University of Alabama. She has experience working with many industries such as automotive, chemical distribution etc. on transportation and operations management projects. She works extensively with food banks and food pantries on supply chain management and logistics focused initiatives. Her graduate and undergraduate students are integral part of her service-learning based logistics classes.

She teaches courses in strategic relationships among industrial distributors and distribution logistics. Her recent research focuses on engineering education and learning sciences with a focus on how to engage students better to prepare their minds for the future. Her other research interests include empirical studies to assess impact of good supply chain practices such as coordinated decision making in stochastic supply chains, handling supply chains during times of crisis and optimizing global supply chains on the financial health of a company. She has published her research in Journal of Business Logistics, International Journal of Physical Distribution and Logistics Management and peer-reviewed proceedings of the American Society for Engineering Education.

\section{Dr. Sharon Lynn Chu, University of Florida}

Dr. Chu received her B.Soc.Sci (1st Class Honors) in Communication and New Media from the National University of Singapore, her MS in Computer Science and Applications and a graduate certificate in Human-Computer Interaction from Virginia Tech, and her Ph.D in Human-Computer Interaction from Texas A\&M University. She is the Director of the the Embodied Learning and Experience Lab at the University of Florida. The ELX Lab conducts research in two main areas: cyberlearning and positive computing.

\section{Dr. Mathew Kuttolamadom, Texas A\&M University}

Dr. Mathew Kuttolamadom is an associate professor in the Department of Engineering Technology \& Industrial Distribution and the Department of Materials Science \& Engineering at Texas A\&M University. He received his Ph.D. in Materials Science \& Engineering from Clemson University's Int'l Center for Automotive Research. His professional experience is in the automotive industry including at the Ford Motor Company. At TAMU, he teaches Mechanics, Manufacturing and Mechanical Design to his students. His research thrusts include bioinspired functionally-graded composites, additive/subtractive manufacturing processes, laser surface texturing, tribology, visuo-haptic VR/AR interfaces and engineering education.

\section{Prof. Francis Quek, Virginia Polytechnic Institute and State University}

Francis Quek is a Professor at Texas A\&M University (Visualization, and Computer Science and Engineering and Psychology - by courtesy). He joined Texas A\&M University as an interdisciplinary President's Signature Hire to bridge disparities in STEM. Formerly he has been the Director of the Center for 
Human-Computer Interaction at Virginia Tech. Francis received both his B.S.E. summa cum laude (1984) and M.S.E. (1984) in electrical engineering from the University of Michigan. He completed his Ph.D. in Computer Science at the same university in 1990. Francis is a member of the IEEE and ACM. He performs research in Human-Computer Interaction (HCI), learning sciences and technology, embodied interaction, embodied learning, HCI for accessibility (especially for individuals with blindness), multimodal verbal/non-verbal interaction, multimodal meeting analysis, vision-based interaction, multimedia databases, medical imaging, assistive technology for the blind, human computer interaction, computer vision, and computer graphics. http://teilab.tamu.edu

\section{Alexander Nicholas Berman, Texas A\&M University}

Alex Berman is a computer science PhD student in TEILab at Texas A\&M University, researching how both pedagogical processes and computational tools can support learning similar to what would occur in STEM-related communities of practice. His research leverages existing online tools and resources, in conjunction with machine learning methods, to help support the learning of 3D printing practices. 


\section{Making in The Colonias: Motivating STEM Participation through a Making as Micro-Manufacturing Model}

\section{Abstract}

Student Making kits and structured projects are designed to encourage Maker based learning in classrooms and organizations. A side effect of these kits is that they abstract the intricacies of the materials in Making. Existing Making kits are challenging for students to understand how their acquired skills may be applied outside of the classroom. We argue that the abstract nature of existing Making kits obscures students perception of how Making is relevant to their everyday experience and future pursuits. ITEST and AISL has a long-standing interest in enhancing Maker-based learning in both formal and informal environments. Together, they partnered together to support innovative models in Maker-based curriculum to support STEM learning and innovation. The product of ITEST and AISL's collaboration was an Early Concept Grant for Exploratory Research (EAGER) that tested the feasibility of coupling Maker concepts with real world concerns in manufacturing and production engineering in high school classrooms. Through this EAGER, we engaged in pilot research on our Making as Micro-Manufacture $\left(\mathrm{M}^{2}\right)$ model, where individuals make things in the scales of tens to hundreds for real-life everyday use. We examined how $\mathrm{M}^{2}$ could be used as a framework for supporting STEM learning, identity, and self efficacy in high school students. In our application of $\mathrm{M}^{2}$, we combined Making, Engineering, and domain knowledge in elementary science as the foundation for a practice based learning career and technology education (CTE) course. We further situated our study within the Colonias, communities situated along the Texas-Mexico border characterized by its rural setting and current state of economic distress.

Students who participated in the CTE were assembled as part of an autonomous Making/Production team that worked under supervision by Texas A\&M University (TAMU) researchers. For three years, TAMU researchers conducted a daily teleconference supported class to teach basic Making and engineering skills. As a motivating scenario, students are tasked to make/produce materials for instructional hands-on activities for elementary school students in the same community. Year 1 of the project focused on familiarizing students with core Maker skills (basic soldering, wire connections, 3D printing) and production engineering concerns (bulk production, supply chains, and inventory management). Year 2 followed a similar procedure as year 1, differing where the students would engage in 6 week-long production schedules where they were expected to prototype, build, package, deliver, and deploy instructional science kits in a local elementary school classroom. Findings from Year 1 and 2 from our study saw increases in the students' own self efficacy in Making and in engineering. Year 3 of the program investigated how experienced participants can support new participants orientation in $\mathrm{M}^{2}$. 'Junior' members, who are new to the class, are provided a survey of knowledge and skills necessary to engage in 
the $\mathrm{M}^{2}$ model. 'Senior' members, who've previously participated, acted as peer-mentors for 'Juniors'. Findings from Year 3 saw an initial rise in 'asked help' and 'intervened help' instances during the earlier stages of the school year but later saw a decrease school year progressed as Junior students master $\mathrm{M}^{2}$ practices through guidance by Seniors. Our work, through this EAGER, demonstrates an approach to providing a situated and scalable curriculum that models practices in real world industries and those that are yet to come.

\section{Introduction}

Making has the potential to expand students' understanding of STEM topics [1], this through direct interaction with physical materials for personalized designs [2]. More-so, Making has the potential to inspire the Maker Mindset in Makers, meaning that they possess a frame of mind that perceives challenges as something to be overcome through individual agency to solve problems [3]. Because of these benefits, there has been a long standing interest of incorporating Making in the classroom. Despite the wide variety of options for classroom Maker kits, they often share a design trait that makes it difficult for students to generalize concepts outside of the classroom. Jenkins and Bogost has referred to this issue as the sandbox effect, in that while students can engage in STEM concepts within the confines of the kits, the moment one attempts to apply said concepts outside of the kit, they aren't as easily applicable to real world applications (e.g., "little Bits" [4] is a kit of electronic connectors that abstract the details such as wire arrangements and sub-components like resistors through enclosures that enable snap-on connections. While it makes electronics accessible to a younger audience, it hides how it works) [5]. We have argued that the abstract nature of these Making kits obscures students perception of how Making is relevant to their everyday experience and future pursuits [6], [7].

ITEST and AISL has a long-standing interest in how Making could support learning in both formal and informal environments. Through their partnership, ITEST and AISL sought to investigate innovative models in Maker-based curricula to better understand how it may suggest a way to support STEM learning and creativity. The product of ITEST and AISL's collaboration was an Early Concept Grant for Exploratory Research (EAGER) that tested the feasibility of coupling Maker concepts with real world concerns in manufacturing and production engineering in high school classrooms. Through the EAGER, we engaged in a 3-year pilot research study on how the Making as Micro-Manufacture $\mathrm{M}^{2}$ model may create a situated learning space. In the $\mathrm{M}^{2}$ model, students can develop self-constructed educational experiences through their direct engagement in Making for everyday, real world use. Practically, we applied the $\mathbf{M}^{2}$ model in the form of a practice-based learning career and technology education (CTE) course focused on combining Making, Engineering, and elementary science curriculum implementation.

For our study, we pursued the following two research questions:

\section{A RQ-I: How effective is the PrBL (practice based learning) model to train students in $\mathbf{M}^{2}$ ?}

B RQ-II: Does engagement in $\mathrm{M}^{2}$ affect STEM knowledge acquisition and self-concept?

Our paper is organized as follows. First, we will provide context on Making through section 2, 'Relevant Background', explaining how our inquiry in this EAGER arose from the intersection of 
Making, manufacturing, and production engineering concerns. Following after, in section 3, 'The $\mathrm{M}^{2}$ Model', we illustrate how our model of combined Making with real-world situatedness and consider its implications for engineering education. Section 4 describes how we applied the $\mathbf{M}^{2}$ Model to an CTE classroom in the Colonias community. We illustrate our findings in section 5, 'Findings'. In section 6, 'Limitations and Future Work', we will review our limitations and point to future work.

\section{Background}

\subsection{Defining Making}

Making refers to the practice of creating tangible objects ranging from sketches to manufactured products [8] that are typically produced within the context of creative communities centered on technology and innovation (e.g., hobbyists and professionals dedicated to making personalized fully functional objects) [9], [10] .

We frame our understanding of Making through the perspective that Making goes beyond the kind of equipment and facilities that are used. Instead, Making is the emergent culture that comes out of the confluence of individuals with shared interests, varied experiences, and a willingness to learn from and impart knowledge towards one's community. Altogether, this summates into the idea of the Maker Mindset [3], a frame of thinking where one believes that he/she has the means to solve any problem faced [11]. Through the Maker Mindset, we believe that students could engage in deeper and personally constructed STEM learning.

While it is recognized that there is a potential for significant STEM learning experiences through Making, existing classroom kits (e.g., LittleBits, Lego Mindstorms) [4] make it difficult to generalize learned concepts beyond the representation shown in class. Essentially, in making the kits themselves accessible or constrained in class, it obfuscates how inherent concepts may appear in real-world scenarios [5].

\subsection{The $\mathbf{M}^{2}$ Model}

Our work has an interest in pushing Making beyond the 'boutique manufacturing' paradigm where products are produced singly and are highly personalized. This manufacturing approach is challenging for repetition and reproduction in volume production. We put forth that the 'Making through Micro Manufacturing' $\left(\mathrm{M}^{2}\right)$ [6], [7] model extends Making with consideration to how supportive technology (e.g., 3D printing machines) may serve to support low-volume manufacturing while affording some level of personalization (Figure 1).

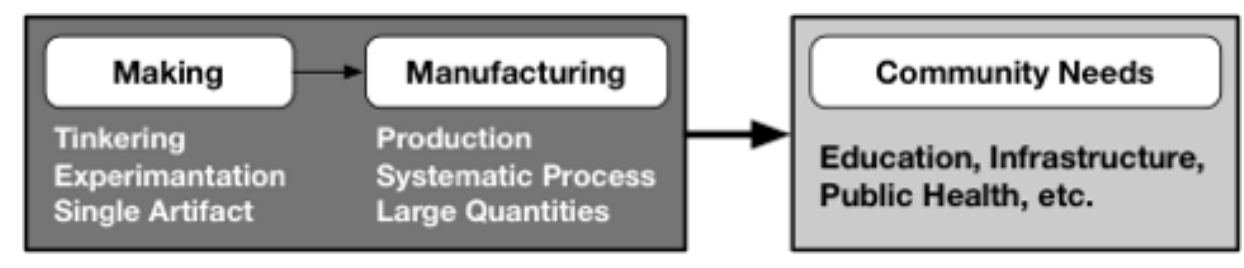

Figure 1: Micro-manufacture model 
Through the $\left(\mathrm{M}^{2}\right)$ model, Making is placed within a real-work context where there are expectations and deadlines associated with a designed products. By situating the practice of Making in such a context, STEM elements become material through its process. We attribute this in how Making can be situated in ways that are personally, socially, and culturally relevant to the student. Second, $\mathbf{M}^{2}$ gives students a sense of agency as they become a part of a team that contributes value to the society. Finally, $\mathrm{M}^{2}$ engages students in long-term scenarios that involve continual thinking and iteration in work.

\section{$4 \mathbf{M}^{2}$ in the Colonias}

In order to evaluate the viability of the $\left(\mathrm{M}^{2}\right)$ model, we needed a real-world scenario to properly motivate it. We found such a scenario through our TAMU's ongoing relationships with the Colonias communities. The Colonias are defined as communities situated along the Texas-Mexico border, characterized by its rural setting and current state of economic distress. The community's school district, Webb CISD, serves its homogeneous population of Hispanic students. Because of the Webb CISD's regional location and economic state, there are fewer tools and access to effectively engage students in STEM education.

After discussion with school officials and identifying STEM interested high school teachers, we developed a collaborative relationship to address the unique context that the Colonias was situated in. In the following subsections we will illustrate how we initialized our $\mathrm{M}^{2}$ centered model in the Colonias. First, we will discuss how we took learned STEM and Maker concepts class and situated them for real-world use. Second, we will illustrate how we modified the program so that it may gain a level of self-sustainability through the community of practice developed from students' experience engaging in $\mathrm{M}^{2}$ (Figure 2).

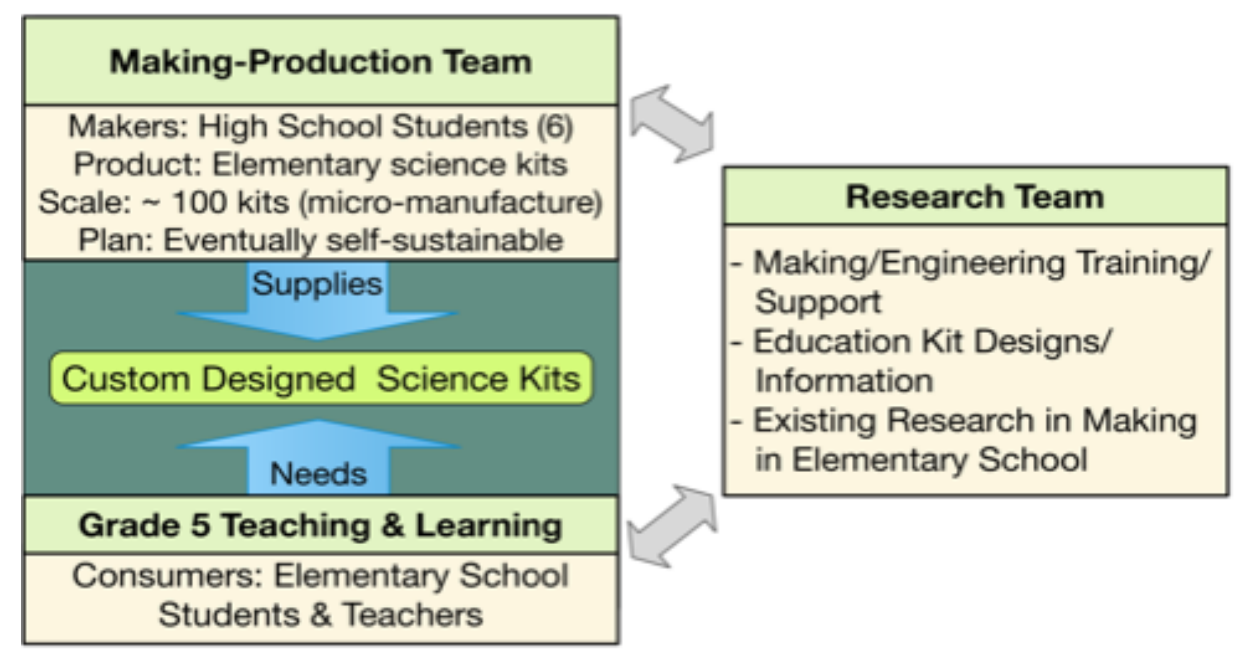

Figure 2: $\mathrm{M}^{2}$ in the Colonias relationships across highschool students, elementary school teacher, and university support team. 


\subsection{Year I}

We first needed to provide a framework for the high school course. Following from our production process for instructional science-kits in the 'Making the Maker' project [12], we formalized the process that was necessary to produce the kits (Figure 3). The intended users of the kits were a 5th grade science class nearby the high school. Through the production process, we generalized the core skills needed to engage in Making. We identified the skills including knowledge of core Making skills (e.g., soldering, wire connections, 3D fabrication), production engineering (e.g., volume production and supply lines), elementary school level science domain knowledge, and design skills. In setting up the course, we provided the partnered high school with a fully furnished Makerspace equipped with a 3D printer, hardware tools, software, computers, and replenishable supplies like wires and solder. In addition, we provided video and audio equipment so that we could record day to day operations of students' activities in the class.

Through the identification of skills, we assembled classroom materials for the high school students in the form of illustrative slides and videos. Given the practicalities of having either a graduate or undergraduate lead the class in person, as far as the high school was from Texas A\&M University (TAMU), we conducted the daily class through video teleconference.

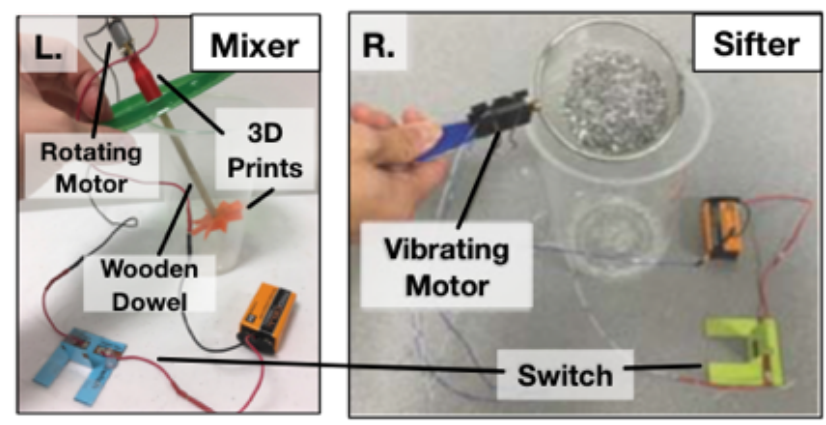

Figure 3: Instructional science kits made by students in CTE.

Before we formally started the class, we hosted a 1-week in-person workshop for the students in the class. We brought the 6 students and their two teachers to TAMU campus. We did this because it was necessary to properly orient the students to Making practices and skills.

We structured the class around the high school's 6-week schedule for each semester. Each week is treated as a separate production pipeline step ranging from initial review of the lesson plan: 1)decomposition of kit parts and ordering of needed parts; 2)creating a minimal viable prototype and designing a production plan; 3)engage in production of kits; 4)packaging kits; 5)deploy kits in the classroom; 6)perform a post-mortem review of strengths and weaknesses of the past six week's production pipeline. As this was occurring, students were surveyed weekly to observe any changes that occurred in their Maker Mindset and self efficacy on math and engineering. Maker Mindset is a scale measure following a 7-point Likert scale using the Maker Mindset Assessment [3]. The assessment consists of 11 items that measures core facets of Making including creativity and teamwork (e.g., "I am willing to help other people", "I like to share things I make with other people”). Student's self-efficacy on Math (e.g., "I feel I am very good at math") and Design (e.g., "Being good at engineering is an important part of who I am"), using an adapted version from the 'Sources of Self Efficacy' scale [13]. 
Daily class operations are characterized as follows. The distant instructor would call in by video teleconference. The students would gather in front of the camera and would participate in a lesson given by the instructor or students would provide an update of their activities of their production and describe their goals for that given day (Figure 4). After this checkup, the students would resume their production activities. During this phase, the students would talk to the distant instructor if they had a question or if the instructor noticed that the students were having difficulty in their tasks (Figure 5).

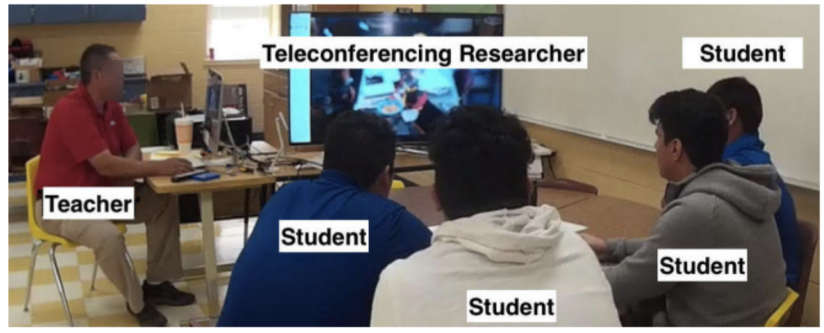

Figure 4: Daily class interactions with teleconference instructor.

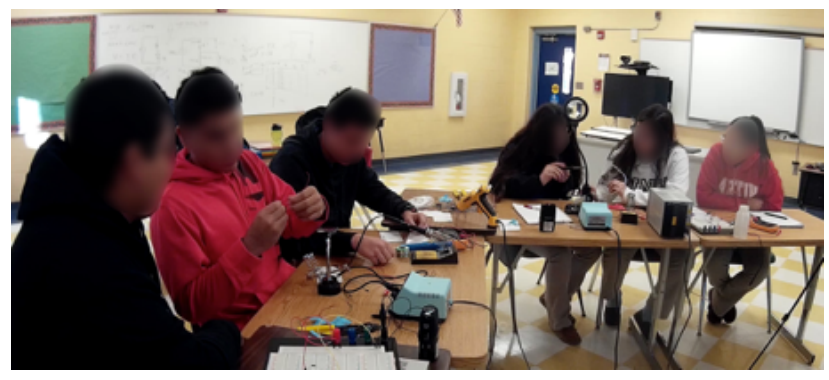

Figure 5: Daily class in process.

\subsection{Year II}

Year II expanded upon concepts that students learned in year I, but differed where students were expected to take the kits that they developed and deploy them for real-world use in elementary school science classes (Figure 2). The students' focus for year 2 is the deployment of the instructional science kits for use in elementary school teachers' classrooms. With this focus, the students needed to develop concern for the teachers' expectations and timeline. Similar to year 1, the students produce the kits in a 6 week production timeline, with a total of 3 kits deployed per semester. The students would contact the elementary school teacher and schedule a time when they could come to her class and deploy the kit for use for the days they are allotted. Unlike year 1 , where the students merely produced the kits from the supplied lesson plans, the students needed to frequently contact the teacher to adapt the kits based on the teachers' own unique needs and end goal.

\subsection{Year III}

Year III resulted in an interest to investigate the feasability of the $\mathrm{M}^{2}$ model to take on self-sustainability as students enter and exit the program. Year III presented a problem of how to 
structure the course as students enter and leave as they move up to the 11th grade or graduate. In order to avoid having to run two separate classes for those experienced or not experienced in Making, we opted for a dual, concurrent section format. 'Junior' students are either 11th grade or 12th grade students who have no prior experience in Making. 'Senior' students are those with prior experience in Making and are likely to have been in 11th grade the year before. Two of the previous students from Year II graduated and two other students had to leave due to schedule conflicts. Two of the remaining students from the original cohort remained while two new students joined the class.

We organized the class as described in Figure 6. Here, Junior students are taught primarily the skills needed to engage in $\mathrm{M}^{2}$, similar to what the previous cohort experienced in Year I. While Junior students are learning core Making skills, they are lead by the Senior students in production. Junior students act under Seniors as their points of execution for any step in the production pipeline. Senior students' were taught skills regarding management, acting as the leaders for various parts of the production cycle. While the students are still taught by the teleconference instructor, the Senior students acted as the point persons for all activities in the classroom.

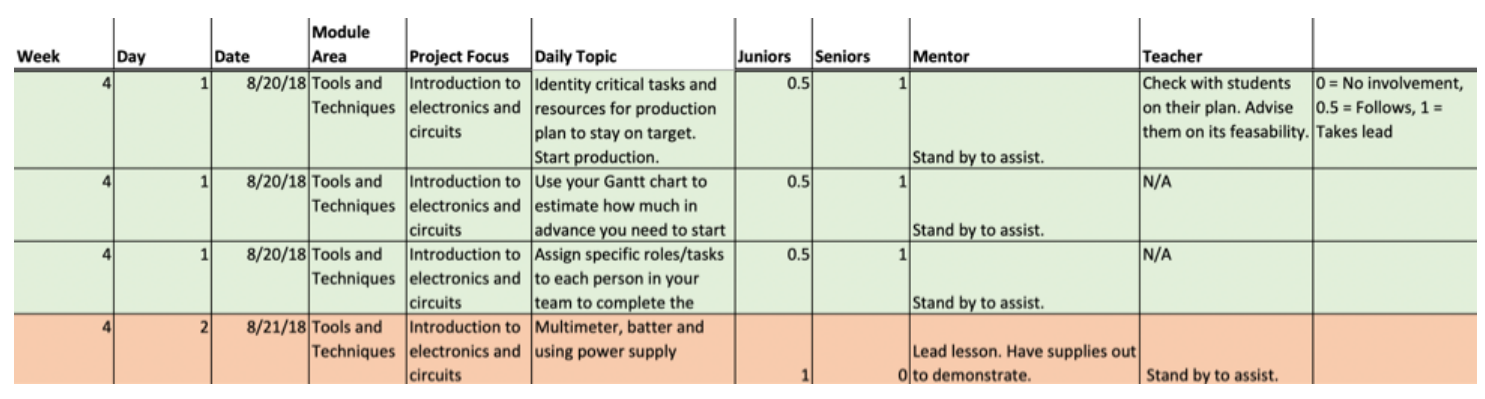

Figure 6: Example Week Using Dual Tiered Schedule

\section{Findings}

\subsection{RQ I: How effective is the PrBL model to train students in $\mathrm{M}^{2}$ ?}

Below we provide two samplings of our findings from the entirety of our study.

\subsubsection{Year I and II Growth for P5:}

Before participating in the our CTE course, P5 had some experience in Making through working with his uncle on small everyday electronics projects like reassembling smart phones or repairing various electronics in their ranch. Owing to P5's experience in electronics, he felt comfortable with taking on the role of project manner for the first production cycle for year 2. During the first year, P5 was quiet and introverted, yet he demonstrated a significant interests towards the technology-centered elements of the production process, often partnering with P4 in these activities. While P5 was comfortable in technology oriented roles, when tasked to take on production management, he was fully engaged in the role for the entirety of the 6 week cycle while still retaining interest in technology when he can. A visualization of his development is shown in figure 7. 


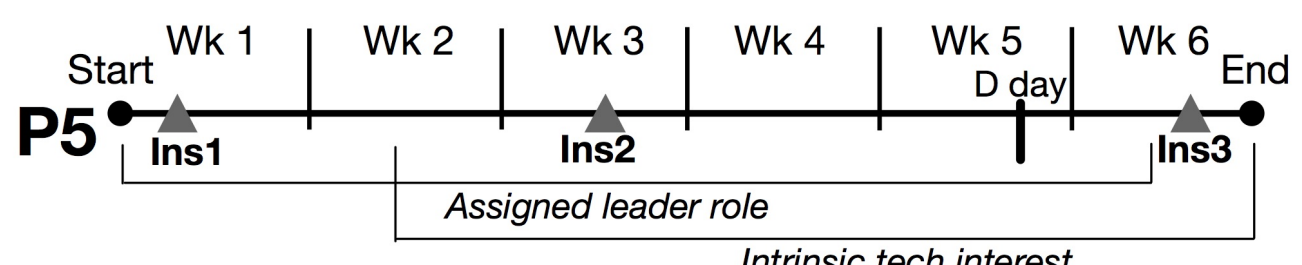

Intrinsic tech interest

Figure 7: P5's six-weeks development

From week 1 to 2, P5 showed leadership traits by acting as the representative for the other students when interacting with daily mentors. An example of this is when P5 was asked how the students were organizing their shared online drive. P5 was the first to speak up and provided a detailed account of the drive. During week 3, between the prototyping and production phase of the cycle, P5 took notice of an issue of a 3D file provided by the mentors. The problem, as P5 told the mentors, would result in a stiff part connection if printed by the classroom's 3D printer. P5 took consideration to the potential delay that might result from a redesign and suggested to the mentors a post-processing approach through sanding Figure 7 (Ins2).

By week 6, after deployment (D day in Wk 5 of 7, we saw P5 once more engage in his interest in technology, where when another student was not sure of how to assemble a battery enclosure for the next kit. P5 took the student aside and explained to her each part in its assembly (Figure 7 (Ins 3)).

\subsubsection{Year III Setting the Stage for a Sustainable Community of Practice:}

Year III focused on how our modified dual-tiered curriculum design could supported a community of practice [14]. The data shown was collected from a 1 month sampling of audio and video recordings. Figure 8 shows the frequency of help that was given and received by different individuals within the class. Teachers and Senior students gave the most help. What is worth noting is that the individual Senior students' help contribution is proportional to half the Teacher's own contribution. In addition, while the TAMU researchers (TR) still provided help, we can see that the senior students provided more help than TR did. Figure 8 also shows where much of the help requested came primarily from the new students that joined the class. We could also see in figure 9 on how help given on each day increased initially then decreased as the production cycle proceeded.

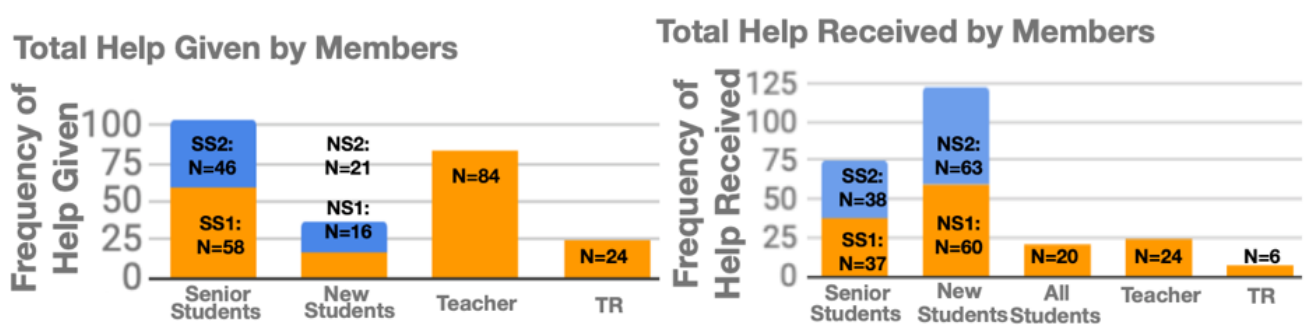

Figure 8: Instances of help given and received across individuals. 


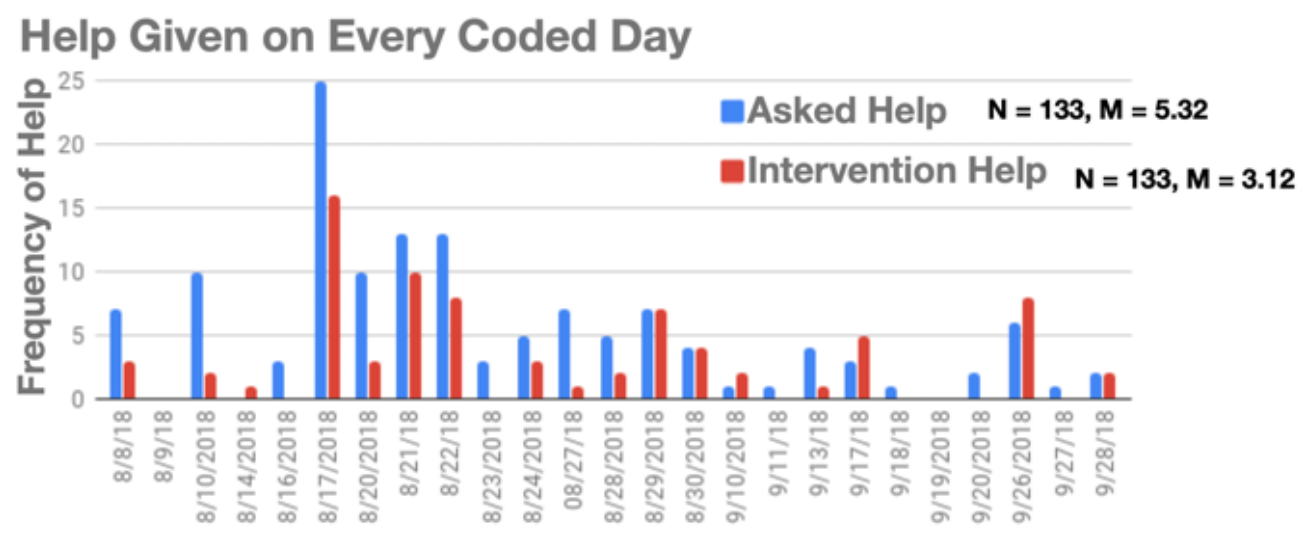

Figure 9: Frequency of help events in class.

What we can draw from these findings is the extent of involvement of the Senior students in daily class interactions. Unlike the Year I, where the initial cohort was just starting to learn the core of M2 and being mentored directly by the university researchers, this same cohort in Year II is now taking charge of the course, acting in many instances as the mentor for the new cohort.

\subsubsection{Year I, Year II, and Year III Implications for $\mathbf{M}^{2}$}

What we can gather from these two samplings from Year I and Year II is that the PrBL model is effective in training students in M3. We saw this first in P5 in expanding his activity range from one of technology orientation to acting as manager, demonstrating a capability to work beyond one's interest area or comfort, signifying a flexibility of mind necessary to attend to both Making and production concerns in M3. Year III extended this finding by demonstrating how the Senior students may act as another point of mentorship in the class for the Junior students, providing an example of how might a community of practice can be formed and sustained as individuals enter and leave the program. Through the M3 model, students can be taught the fundamental practices of coupling Making and production engineering, practice and improve upon said skills through real-world application, and further solidify their understanding by passing their uniquely formed knowledge to others as the program progresses.

\subsection{RQ-II: Does engagement in $\mathrm{M}^{2}$ affect STEM knowledge acquisition and self-concept?}

In the interest of studying any changes in STEM knowledge and self-concept through participation in the program, we ran three separate Wilcoxon signed-rank tests. The tests were based on the student's $(\mathrm{N}=6)$ pre-and-post scores on Maker Mindset and self efficacy for math and engineering. Pre and post-tests efficacy comparison showed a statistically significant difference between the pre and post score for Maker Mindset $(Z=1, p=0.12)$ and math self efficacy $(Z=4, p=0.028)$ (Figure 10). The pre-test reports students believed that it was true that they possessed a Maker Mindset and that it was somewhat true that they believed they were capable in math. By the post test, students then reported that it was somewhat true that they had the Maker Mindset and true that they were capable in Math. We saw no statistically significant difference between pre-and-post scores for engineering self efficacy. 
While the students demonstrated a reported increase in Math self efficacy through engaging $\mathrm{M}^{2}$, we saw no such increase in engineering self efficacy. This could be attributed to the how the course was set out as it was the university support team that provided the lesson plans and kit designs to the students and not the students. We also saw a slight decrease in Maker Mindset between year I and year II. Year II was differentiated from year I where the students actually had to deploy the kits in the classroom, rather than engage in practice in setting up the kits from the previous year. It is likely that when the students were faced with the demands and pressures that comes with actual classroom deployment, they had to reassess their skills in order to ensure a quality end-product.

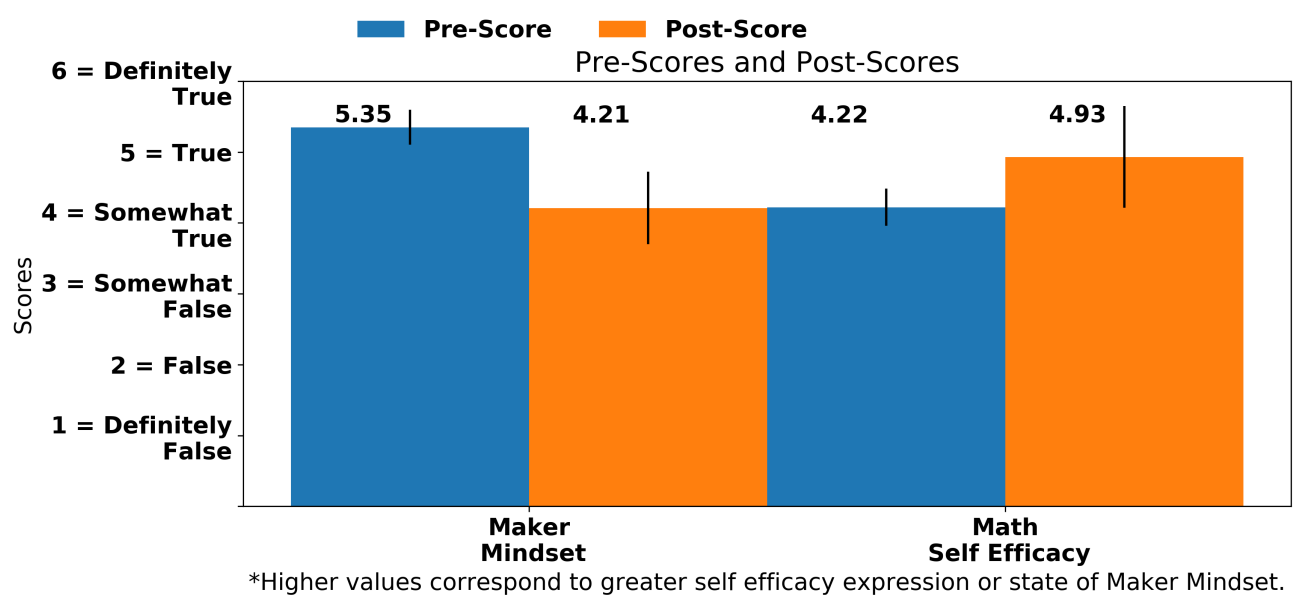

Figure 10: Pre and post score comparison of Maker Mindset and Math Self Efficacy.

\section{Limitations and Future Work}

Our $\mathrm{M}^{2}$ program demonstrated how Making could be situated in the classroom through the coupling of self-constructed educational experiences that comes with Making alongside developed skills for the production of instructional science kits for real world end-use by elementary school classrooms. We recognize that this study does not provide the definitive answer of how Making can be made effective in the classroom, but rather, is an opening inquiry of a future line of research. Here, we will identify areas of future work that we will take our line of research in $\mathbf{M}^{2}$.

First, we would like to consider the role of setting. Our study was conducted in the Colonias region, which contains its own unique cultural and social context as a rural center. We would like to compare how such an implementation of $\mathrm{M}^{2}$ may prove similar or different across different rural settings, so that we may develop a better understanding of how $\mathrm{M}^{2}$ addresses inherent STEM participation issues common to rural areas. In addition, we would like to examine how $\mathrm{M}^{2}$ may support other types of communities across different settings across regional and economic lines so that we may understand how might $\mathrm{M}^{2}$ afford flexibility in its sensitivity to the context of a given educational setting.

Second is further consideration to the medium of how class was supported. As we mentioned 
previously, the Colonias $\mathrm{M}^{2}$ course was supported through a daily teleconference class sessions lead by an distant undergraduate/graduate student instructor. Through the course of the study, we found certain challenges that came with supporting the inherently hands-on physical content that Making is based on. When lacking a physical body to interact with students, both in terms of practical actions in handling materials and subtle interactions, such as how physical explanations through hand gestures communicate subtle details, we found that there were disconnects in how class proceeded. Following from this insight, we are continuing our work with the Colonias community, bringing in telepresence robotics to investigate how simulated physical embodiment may serve to bridge a gap in students' experiential and learning outcomes in Making and STEM learning in distance education [15]. In addition, we will conduct controlled studies that compare across in-person instructor, teleconference, and telepresence robot modalities.

\section{Conclusions}

The potential for Making to support STEM learning for students has been widely recognized but prior attempts to situating Making in the classroom has resulted in end-products that make it difficult to generalize learned concepts outside of the classroom. We argued that if Making was coupled with the concerns of real-world practice such as real-world use, deadlines, and appropriate volume, the STEM concepts inherent in Making could become all the more apparent in students' Making experience. To evaluate this concept, we partnered with a school district situated in the Colonias, creating a three year program that provided STEM learning opportunities for the community. In year 1, we found that students developed significantly greater self-efficacy outcomes across engineering, design, and Making. Year 2 followed the same group of students and saw how they took their knowledge and applied it in real-world classrooms as they deployed their built instructional science kits. Here, students learned how to optimize their resources when given expectations for real-world use. Finally, in year 3, we saw how as cohorts of students enter and leave, those left in the intermediary serve as a bridge of continuity, mentoring junior students in the practices of $\mathrm{M}^{2}$. Future work will investigate how the communication medium, currently video teleconferencing, may be further augmented via telepresence robotics to aid the distant instructor in embodied practices such a maintain gaze and engaging in physical explanations.

\section{References}

[1] E. R. Halverson and K. Sheridan, "The maker movement in education," Harvard Educational Review, vol. 84, no. 4, pp. 495-504, 2014.

[2] P. Blikstein, "Digital fabrication and 'making' in education: The democratization of invention," FabLabs: Of machines, makers and inventors, vol. 4, pp. 1-21, 2013.

[3] T. M. E. Foundation, "The maker mindset assessment," 2015.

[4] A. Bdeir, "Electronics as material: littlebits," in Proceedings of the 3rd International Conference on Tangible and Embedded Interaction, pp. 397-400, ACM. 
[5] T. Jenkins and I. Bogost, "Escaping the sandbox: Making and its future," in Proceedings of the Ninth International Conference on Tangible, Embedded, and Embodied Interaction, pp. 29-32, 2015.

[6] O. J. Okundaye Jr, M. Kuttolamadom, M. Natarajarathinam, S. L. Chu, and F. Quek, "Motivating stem participation through a'making as micro-manufacture (m3)'model," in 2018 ASEE Annual Conference Exposition.

[7] O. Okundaye, S. Chu, F. Quek, A. Berman, M. Natarajarathinam, and M. Kuttolamadom, "From making to micro-manufacture: Catalyzing stem participation in rural high schools," in Proceedings of the Conference on Creativity and Making in Education, pp. 21-29, ACM.

[8] e. a. Hlubinka, M., "Makerspace playbook: School edition," 2013.

[9] D. Kera, "Hackerspaces and diybio in asia: connecting science and community with open data, kits and protocols," Journal of Peer Production, vol. 2, no. Jun, pp. 1-8, 2012.

[10] M. L., "What is maker culture? - diy roots," 2009.

[11] M. Honey and D. Kanter, "Design, make, play: growing the next generation of science innovations," Rutledge, London, 2013.

[12] e. a. Quek, F., "Making the maker: A pathway to stem for elementary school students," 2014.

[13] E. L. Usher and F. Pajares, "Sources of self-efficacy in mathematics: A validation study," Contemporary educational psychology, vol. 34, no. 1, pp. 89-101, 2009.

[14] M. O. J. Okundaye Jr, "Developing communities of practice through peer mentorship in making through micro-manufacturing model,"

[15] O. Okundaye, F. Quek, and S. Chu, "Broadening participation for remote communities: Situated distance telepresence," in Proceedings of the 18th ACM International Conference on Interaction Design and Children, pp. 494-500, 2019. 